\title{
Solar and atmospheric neutrino mass splitting with SMASH model
}

\author{
C.R. Das ${ }^{1}$, Katri Huitu ${ }^{2}$, Timo Kärkkäinen ${ }^{3}$ \\ ${ }^{1}$ Bogoliubov Laboratory of Theoretical Physics, Joint Institute for Nuclear Research, \\ Joliot-Curie 6, Dubna 141980, Moscow region, Russian Federation \\ ${ }^{2}$ Helsinki Institute of Physics, P.O.Box 64 (Gustaf Hällströmin katu 2), \\ FI-00014 University of Helsinki, Finland \\ ${ }^{3}$ Department of Physics, P.O. Box 64 (Gustaf Hällströmin katu 2), \\ FI-00014 University of Helsinki, Finland \\ E-mail: ${ }^{1}$ das@theor.jinr.ru, ${ }^{2}$ katri.huitu@helsinki.fi, ${ }^{3}$ timo.j.karkkainen@helsinki.fi
}

\begin{abstract}
Five fundamental problems - neutrino mass, baryogenesis, dark matter, inflation, strong CP problem - are solved at one stroke in a model, dubbed as "SM-A-S-H" (Standard Model-Axion-Seesaw-Higgs portal inflation) by Andreas Ringwald et. al. The Standard Model (SM) particle content is extended by three right-handed SM-singlet neutrinos $N_{i}$, a vector-like color triplet quark $Q$, a complex SM-singlet scalar field $\sigma$ that stabilises the Higgs potential, all of them being charged under Peccei-Quinn (PQ) $U(1)$ symmetry, the vacuum expectation value $v_{\sigma} \sim 10^{11} \mathrm{GeV}$ breaks the lepton number and the Peccei-Quinn symmetry simultaneously. We found that numerically SMASH model not only solves five fundamental problems but also the sixth problem "Vacuum Metastability" through the extended scalar sector and can predict approximately correct atmospheric neutrino mass splitting around $0.05 \mathrm{eV}$ and the solar neutrino mass splitting around $0.009 \mathrm{eV}$.
\end{abstract}

Keywords: Neutrino mass; Planck neutrino mass limit; Higgs metastability; SMASH model

\section{Introduction}

After the discovery of the Standard Model (SM) Higgs boson [1, 2, 3], every elementary particle of the SM has been confirmed to exist. Even though the last forty years have been a spectacular triumph for the SM, the mass of the Higgs boson $\left(m_{h}=125.09 \pm 0.32 \mathrm{GeV}\right)$ poses a serious problem for the SM. It is well-known that the SM Higgs potential is metastable [4], as the sign of the quartic coupling, $\lambda_{H}$ turns negative at instability scale $\Lambda_{\mathrm{IS}} \sim 10^{11} \mathrm{GeV}$. The largest uncertainties of SM vacuum stability are driven by top quark pole mass and the mass of SM Higgs boson. Present experimental data is in significant tension with the stability hypothesis, making it more likely that the universe is in a metastable vacuum state. The expected lifetime of vacuum decay to a stable vacuum is extraordinarily long, and it is unlikely to affect the evolution of the universe. However, it is unclear why the vacuum state entered to metastable or unstable vacuum, to begin with during the early universe. On this post-SM era, the emergence of vacuum stability problem (among many others) forces the particle theorists to expand the SM in such a way that the $\lambda_{H}$ will stay positive during the running all the way up to the Planck scale, $M_{P}=1.22 \times 10^{19} \mathrm{GeV}$. 
It is possible that at or below the instability scale heavy degrees of freedom originating from a theory beyond the SM start to alter the running of the SM parameters of renormalization group equations (RGE). This approach aims to solve the vacuum stability problem by proving that the universe is currently in a stable vacuum. One theory candidate is a complex singlet $\sigma$ extended SM. The scalar sector of such a theory may stabilise the theory with a threshold mechanism $[5,6]$. The effective SM Higgs coupling gains a positive correction $\delta \equiv \lambda_{H \sigma}^{2} / \lambda_{\sigma}$ at $m_{\sigma}$, where $\lambda_{H \sigma}$ is the Higgs doublet-singlet portal coupling and $\lambda_{\sigma}$ is the quartic coupling of $\sigma$.

This threshold mechanism is embedded in a recent SMASH [7] theory, which utilizes it at $\lambda_{H \sigma} \sim-10^{-6}$ and $\lambda_{\sigma} \sim 10^{-10}$. The mechanism turns out to be dominant unless the new Yukawa couplings of SMASH are $O(1)$.

\section{Theory}

The Standard model one-loop beta function for Higgs potential parameter $\mu_{H}$ is

$$
\frac{\partial \mu_{H}^{2}}{\partial \lg \mu}=\frac{\mu_{H}^{2}}{8 \pi^{2}}\left(-\frac{3}{4} g_{1}^{2}-\frac{9}{4} g_{2}^{2}+3 y_{t}^{2}+3 y_{b}^{2}+y_{\tau}^{2}+3 \lambda_{H}\right),
$$

where light degrees of freedom are ignored and lg denotes the Briggsian logarithm.

One-loop beta function for $\lambda_{H}$ is

$$
\frac{\partial \lambda_{H}}{\partial \lg \mu}=\frac{1}{16 \pi^{2}}\left(12 \lambda_{H}^{2}-\left(\frac{9}{5} g_{1}^{2}+9 g_{2}^{2}\right) \lambda_{H}+\frac{9}{4}\left(\frac{3}{25} g_{1}^{4}+\frac{2}{5} g_{1}^{2} g_{2}^{2}+g_{2}^{4}\right)+4 Y_{2} \lambda_{H}-4 h\right) .
$$

SMASH framework [7] expands the scalar sector of the SM by introducing a complex singlet field $\sigma$. The scalar potential of SMASH is then

$$
V(H, \sigma)=\lambda_{H}\left(H^{\dagger} H-\frac{v^{2}}{2}\right)^{2}+\lambda_{\sigma}\left(|\sigma|^{2}-\frac{v_{\sigma}^{2}}{2}\right)^{2}+2 \lambda_{H \sigma}\left(H^{\dagger} H-\frac{v^{2}}{2}\right)\left(|\sigma|^{2}-\frac{v_{\sigma}^{2}}{2}\right) .
$$

Defining $\phi_{1}=H$ and $\phi_{2}=\sigma$, scalar mass matrix of this potential is

$$
\left(M_{i j}\right)=\left.\frac{1}{2} \frac{\partial^{2} V}{\partial \phi_{i} \partial \phi_{j}}\right|_{\substack{H=v / \sqrt{2} \\
\sigma=v_{\sigma} / \sqrt{2}}}=\left(\begin{array}{cc}
2 \lambda_{H} v^{2} & 2 \lambda_{H \sigma} v v_{\sigma} \\
2 \lambda_{H \sigma} v v_{\sigma} & 2 \lambda_{\sigma} v_{\sigma}^{2}
\end{array}\right),
$$

which has eigenvalues

$$
m_{H}^{2} \approx 2\left(\lambda_{H} v^{2}+\lambda_{H \sigma} v_{\sigma}^{2}\right) \text { and } m_{\sigma}^{2} \approx 2\left(\lambda_{\sigma} v_{\sigma}^{2}+\lambda_{H \sigma} v^{2}\right) .
$$

\subsection{Threshold correction}

Consider an energy scale below $m_{\sigma}<\Lambda_{\mathrm{IS}}$, where the heavy scalar $\sigma$ is integrated out. The low-energy Higgs potential should match the SM Higgs potential:

$$
V(H)=\lambda_{H}^{\mathrm{SM}}\left(H^{\dagger} H-\frac{v^{2}}{2}\right)^{2} .
$$

It turns out that the quartic coupling we measure has an additional term:

$$
\lambda_{H}^{\mathrm{SM}}=\lambda_{H}-\lambda_{H \sigma}^{2} / \lambda_{\sigma} .
$$

Since the SM quartic coupling will be approximately -0.08 at $M_{P}$, the threshold correction

$$
\delta \equiv \frac{\lambda_{H \sigma}^{2}}{\lambda_{\sigma}}
$$

should be large enough to push the high-energy counterpart $\lambda_{H}$ to positive value all the way up to $M_{P}$. In the literature there are two possible ways of implementing this threshold mechanism. 
(i) One may start by solving the SM RGE's up to $m_{\sigma}$, where the SMASH effects kick in, and the quartic coupling $\lambda_{H}$ gains a sudden increment by $\delta$. Continuation of RGE analysis then requires utilizing SMASH RGE's up to the Planck scale.

(ii) Another way is to solve the SMASH RGE's from the SM scale, not bothering to solve the low-energy SM RGE's at all.

We have choosed both approaches respectively in Fig. 1 and 2.

\section{Methods}

The authors of [7] have generated the two-loop $\beta$ functions of SMASH. We solve numerically the 14 coupled renormalization group differential equations with respect to Yukawa $\left(y_{t}, y_{b}, y_{\tau}, y_{F}, y_{N}, Y_{Q}\right)$, gauge $\left(g_{1}, g_{2}, g_{3}\right)$ and scalar couplings $\left(\mu_{H}^{2}, \mu_{S}^{2}, \lambda_{H}, \lambda_{\sigma}, \lambda_{H \sigma}\right)$, ignoring the light SM degrees of freedom, from $M_{Z}$ to Planck scale. We use MATLAB's ode45-solver. See Table 1 for used SMASH benchmark points, and Table 2 for our SM input. Our scale convention is $t \equiv \log _{10} \mu / \mathrm{GeV}$.

In some papers, the running of SM parameters $\left(y_{t}, y_{b}, y_{\tau}, g_{1}, g_{2}, g_{3}, \mu_{H}^{2}, \lambda_{H}\right)$ obeys the SM RGE's without corrections from SMASH until $\mu=\sqrt{2} \mu_{S}$, after which $\lambda_{H}$ gains a threshold correction and the running of all SM parameters follows the SMASH RGE's. We choose a different approach, where we utilize the SMASH RGE's from the beginning to all of the parameters.

We scanned over the parameter space $m_{t} \in[164,182] \mathrm{GeV}$ and $m_{h} \in[110,140] \mathrm{GeV}$. For every point, we investigated the running of the quartic couplings of the scalar potential. If either $\lambda_{H}$ or $\lambda_{\sigma}$ turn negative, we denote this point metastable in Fig. 3. If any of the quartic couplings

$$
1000 \mathrm{~m}_{\mathrm{N}}=\mathrm{v}_{\sigma}=1 \mathrm{e}+09 \mathrm{GeV}, \lambda_{\mathrm{S}}=2 \mathrm{e}-10, \lambda_{\mathrm{HS}}=-3.5 \mathrm{e}-06, \mathrm{Y}_{\mathrm{F}}=0.001, \mathrm{Y}_{\mathrm{Q}}=0.001
$$
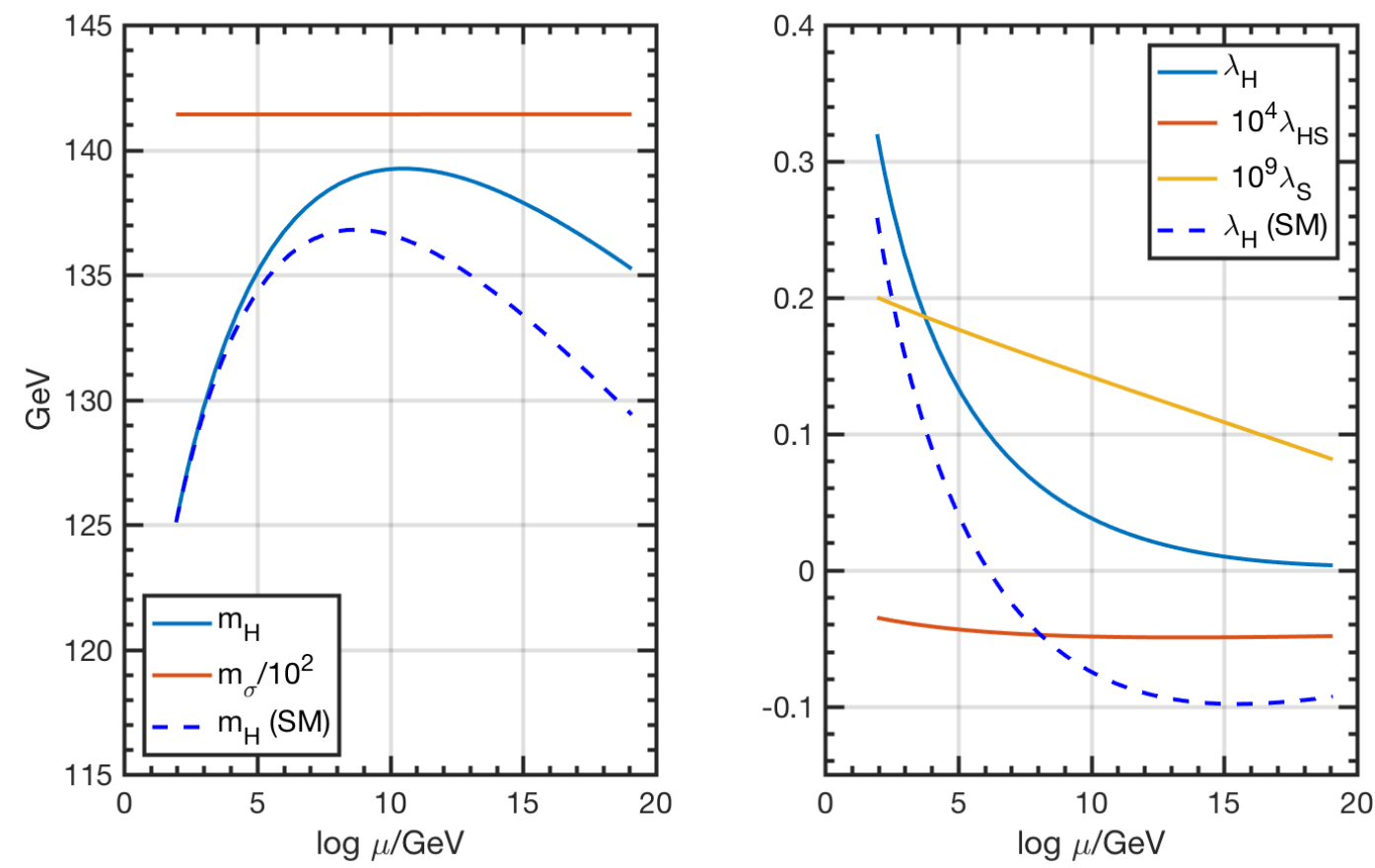

Figure 1. Running of Higgs, $\sigma$ bare mass and scalar potential parameters with benchmark point. Threshold applied from the beginning at $m_{Z}$. 


$$
1000 m_{N}=v_{\sigma}=1 e+09 \mathrm{GeV}, \lambda_{S}=2 e-10, \lambda_{H S}=-4.7 e-06, Y_{F}=0.001, Y_{Q}=0.001
$$
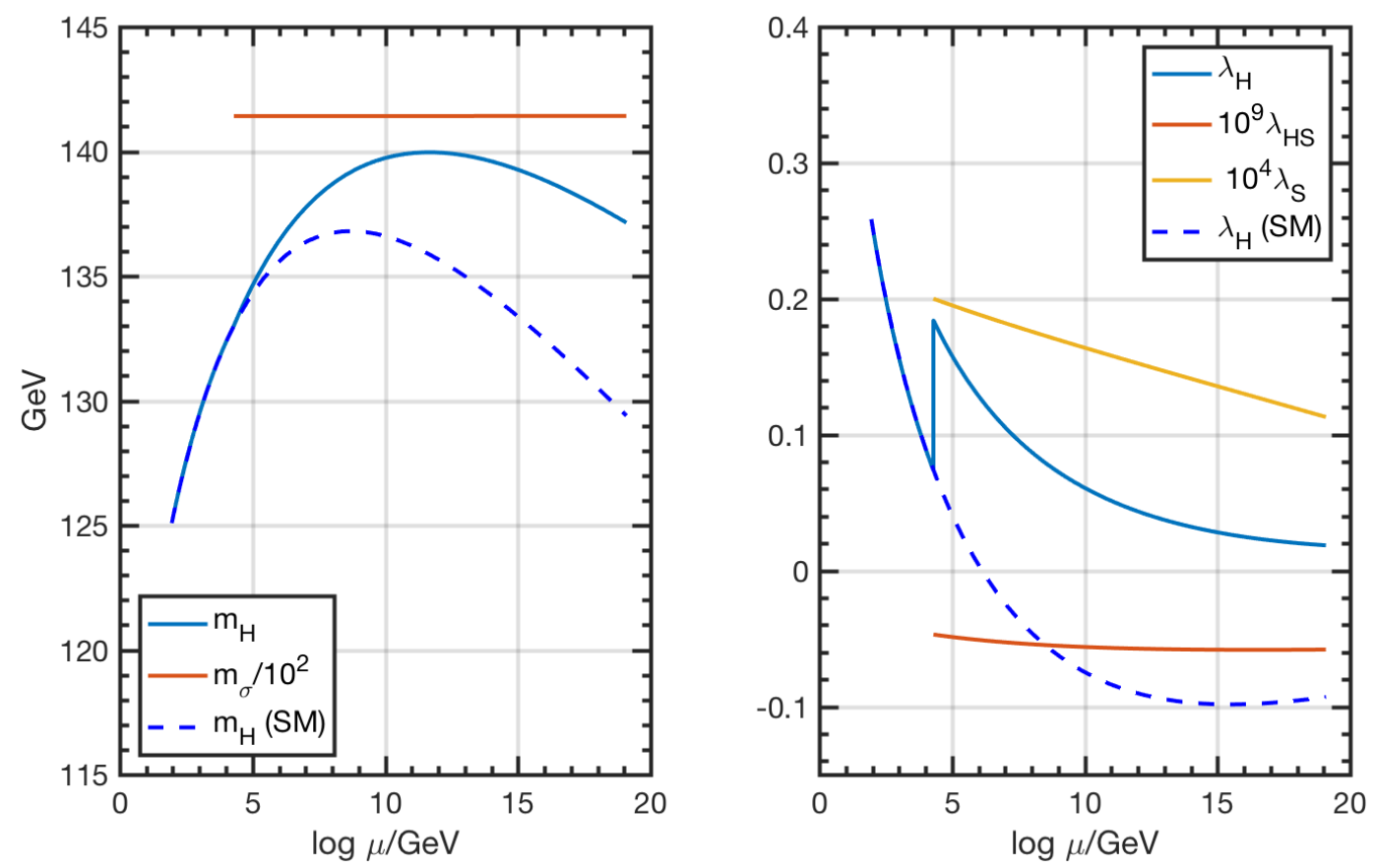

Figure 2. Running of Higgs, $\sigma$ bare mass and scalar potential parameters with benchmark point. Threshold correction utilized at $m_{\rho}$.

Table 1. Used benchmark points (BPs) in our analysis. Note that we assume specific texture to RH and LH neutrino Yukawa matrices $Y_{N}=y_{N} \times \operatorname{diag}(1,2,2.1)$ and $Y_{F}=y_{F} \times I_{3}$, respectively, where $y_{N}$ and $y_{F}$ are numbers.

\begin{tabular}{ccccc}
\hline Parameters & BP1 & BP2 & BP3 & BP4 \\
\hline$y_{F}$ & $10^{-3}$ & $10^{-2}$ & $10^{-3}$ & $3 \times 10^{-3}$ \\
$y_{N}$ & $\sqrt{2} / 25$ & $\sqrt{2} / 1000$ & $\sqrt{2} / 1000$ & $\sqrt{2} / 1000$ \\
$Y_{Q}$ & 0.1 & $10^{-2}$ & $10^{-3}$ & $10^{-3}$ \\
$v_{\sigma}$ & $10^{9} \mathrm{GeV}$ & $10^{8} \mathrm{GeV}$ & $10^{9} \mathrm{GeV}$ & $10^{9} \mathrm{GeV}$ \\
$\lambda_{\sigma}$ & $2 \times 10^{-9}$ & $2 \times 10^{-9}$ & $10^{-10}$ & $1.5 \times 10^{-10}$ \\
$\lambda_{H \sigma}$ & $-10^{-5}$ & $-10^{-5}$ & $-3 \times 10^{-6}$ & $-3 \times 10^{-6}$ \\
$q$ & $-1 / 3$ & $-1 / 3$ & $-1 / 3$ & $-1 / 3$ \\
\hline
\end{tabular}

rises above unity, we denote this point non-perturbative. Analytically a small value of $\lambda_{H \sigma}$ can give positive correction at the one-loop level to push $\lambda_{H}$ out of the valley of instability, and the correlations of other SMASH parameters to $\lambda_{H}$ are small.

\subsection{Choice of benchmark points}

To avoid the overproduction of dark radiation via the cosmic axion background, we choose $\lambda_{H \sigma}<0$. To obtain the observed matter-antimatter asymmetry via leptogenesis, a hierarchy 
Table 2. Used SM inputs in our analysis, at $\mu=m_{Z}=91.18 \mathrm{GeV}$. We define $\mu_{H}=m_{h} / \sqrt{2}$ and $\lambda_{H}=m_{h}^{2} / 2 v^{2}$.

\begin{tabular}{cl||cl}
\hline Parameters & Values & Parameters & Values \\
\hline$m_{t}$ & $173.1 \mathrm{GeV}$ & $v$ & $246.22 \mathrm{GeV}$ \\
$m_{b}$ & $4.18 \mathrm{GeV}$ & $g_{1}$ & 0.357 \\
$m_{h}$ & $125.18 \mathrm{GeV}$ & $g_{2}$ & 0.652 \\
$m_{\tau}$ & $1.777 \mathrm{GeV}$ & $g_{3}$ & 1.221 \\
\hline
\end{tabular}

on heavy Majorana neutrinos $N_{i}$ is required. This is achieved by assigning the right-handed Majorana Yukawa matrix a texture: $Y_{N}=y_{N} \times \operatorname{diag}(1,2,2.1)$. As the light neutrinos are also not mass degenerate, we assign the Dirac Yukawa matrix the following texture: $Y_{F}=y_{F} \times \operatorname{diag}(1,1,1)$. Values for $y_{N}$ and $y_{F}$ are in Table 1.

\subsection{Partial unification}

The viable parameter space where the SMASH vacuum is stable turns out to give arise of partial gauge sector unification, where $g_{2}\left(M_{P}\right) \approx g_{3}\left(M_{P}\right)$.

\subsection{Neutrino mass splitting}

Basic version of SMASH utilizes Type-I see-saw mechanism. The mass matrix is

$$
\begin{gathered}
M_{\nu}=\left(\begin{array}{cc}
0 & M_{D} \\
M_{D}^{T} & M_{M}
\end{array}\right)=\frac{1}{\sqrt{2}}\left(\begin{array}{cc}
0 & Y_{F} v \\
Y_{F}^{T} v & Y_{N} v_{\sigma}
\end{array}\right) \\
\text { and } m_{\nu}=-M_{D} M_{M}^{-1} M_{D}^{T} \approx-\frac{4 \times 10^{8} \mathrm{GeV}}{v_{\sigma}} \times \frac{Y_{F} Y_{N}^{-1} Y_{F}^{T}}{10^{-6}} \times 10^{-2} \mathrm{eV} .
\end{gathered}
$$

Vanilla leptogenesis scenario requires the existence of heavy RH neutrinos, which are too unstable for the dark-matter candidate. The see-saw scale intermediate between SM and GUT scales, slides well into SMASH framework, with the RH neutrino mass given by VEV of $\sigma$.
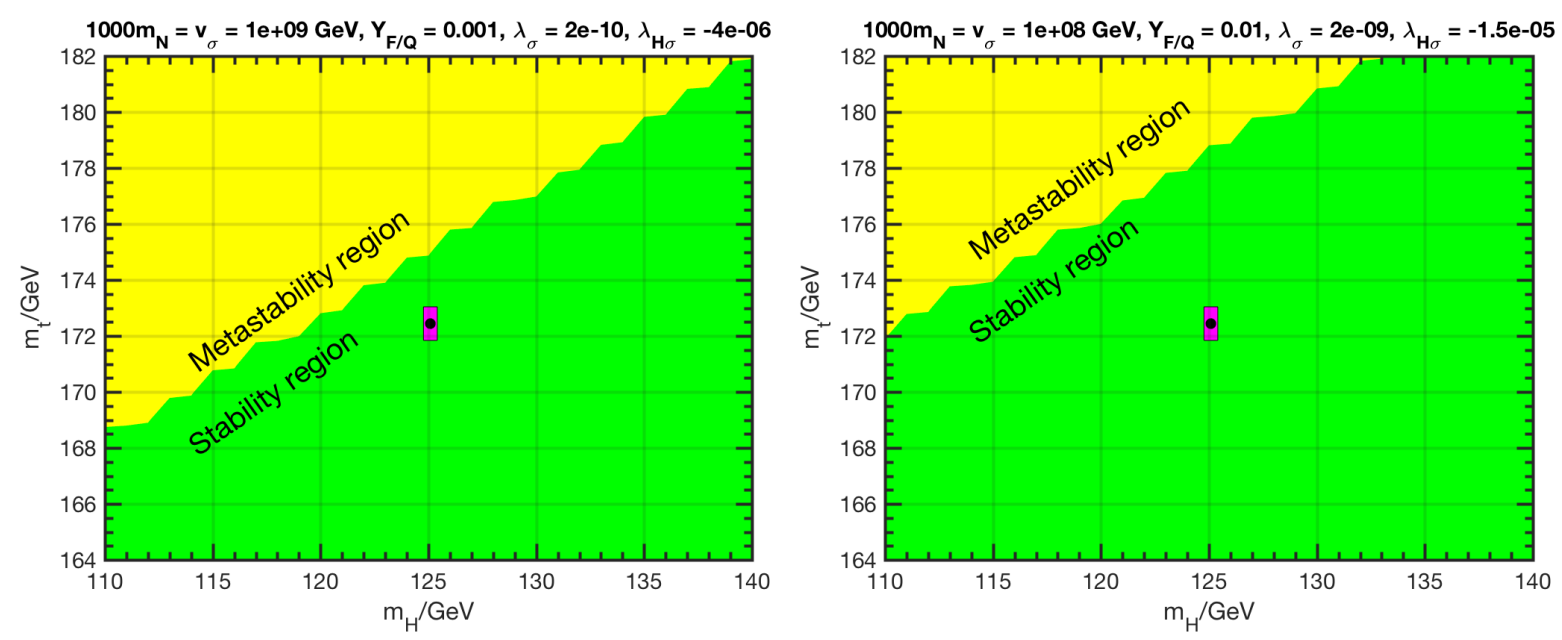

Figure 3. Scalar potential vacuum stability regions for $\lambda_{H \sigma} \approx-10^{-5}$ in $\left(m_{H}, m_{t}\right)$ plane. 
Recent results from PLANCK constraints on sum of neutrino masses to be $<0.12 \mathrm{eV}$ (95 \% CL.). With the known mass splittings known from oscillation experiments, the heaviest neutrino can be at most $\approx 59 \mathrm{meV}$. With a simple Yukawa texture, we are able to fit the present constraints on neutrino masses, see Fig. 4.

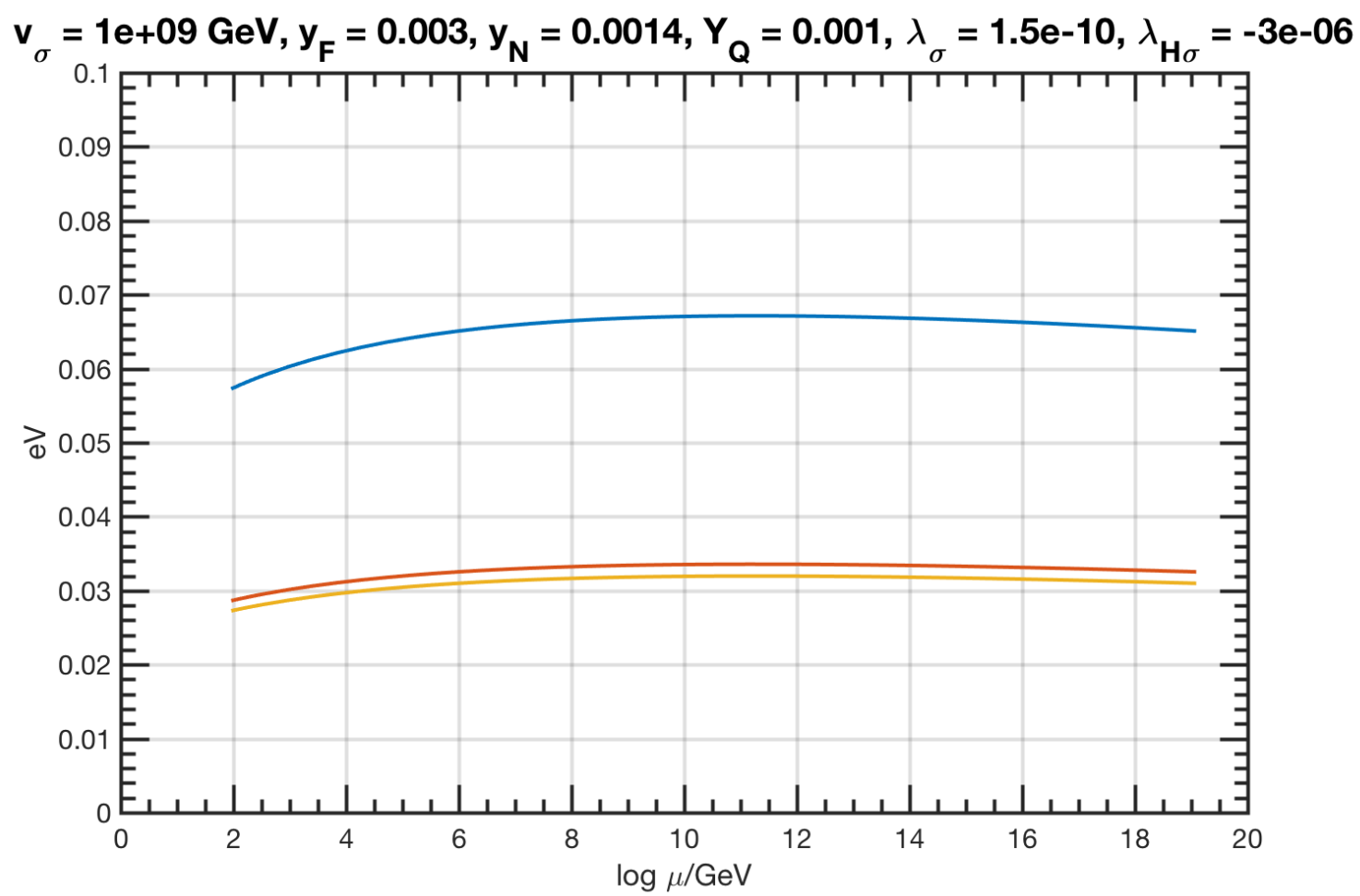

Figure 4. Running of the neutrino masses (mass eigenstates). Mass splitting for atmospheric and solar neutrinos correspond well to present experimental data from oscillation experiments and PLANCK bounds on the sum of light neutrino masses.

\section{Conclusions}

(i) SMASH unifies axions, see-saw and extended Higgs sector on one energy scale, $\mu \sim 10^{10}$ $10^{11} \mathrm{GeV}$, solving several problems badgering the Standard Model in one go.

(ii) SM vacuum is metastable, since $\lambda_{H}$ turns negative around $\mu \simeq 10^{12} \mathrm{GeV}$, SMASH can fix this vacuum metastability problem with $\lambda_{H \sigma} \gtrsim-10^{-5}$ at two-loop RGE level.

(iii) Given a suitable neutrino Yukawa texture, SMASH predicts the correct values to atmospheric neutrino mass splitting $\Delta m_{31}^{2}$ and the solar neutrino mass splitting $\Delta m_{21}^{2}$.

\section{References}

[1] ATLAS Collaboration 2012 Phys. Lett. B 7161 (Preprint arXiv:1207.7214)

[2] CMS Collaboration 2012 Phys. Lett. B 71630 (Preprint arXiv:1207.7235), ibid. J. High Energy Phys. JHEP06(2013)081 (Preprint arXiv:1303.4571)

[3] CDF Collaboration 2013 Phys. Rev. D 88052014 (Preprint arXiv:1303.6346)

[4] Alekhin S, Djouadi A and Moch S 2012 Phys. Lett. B 716214 (Preprint arXiv:1207.0980)

[5] Elias-Miró J, Espinosa J R, Giudice G F, Lee H M and Strumia A J. High Energy Phys. JHEP06(2012)031 (Preprint arXiv:1203.0237)

[6] Lebedev O 2012 Eur. Phys. J. C 722058 (Preprint arXiv:1203.0156)

[7] Ballesteros G, Redondo J, Ringwald A and Tamarit C J. Cosmol. Astropart. Phys. JCAP08(2017)001 (Preprint arXiv:1610.01639), ibid. 2017 Phys. Rev. Lett. 118071802 (Preprint arXiv:1608.05414) 